\title{
A real-time RT-PCR assay for rapid detection of coxsackievirus A10
}

\author{
C.Y. Mu ${ }^{1,4 *}$, A.Y. Wang ${ }^{2 *}$, C. Chen ${ }^{4}$, L. Zhao ${ }^{3}$ and Z. $\mathrm{Li}^{1}$ \\ ${ }^{1}$ Department of Microbiology, Anhui Medical University, Hefei, China \\ 2Department of Clinical Laboratory, First Affiliated Hospital of Anhui Medical University, \\ Hefei, China \\ ${ }^{3}$ Fuyang City Center for Disease Control and Prevention, Fuyang, Anhui Province, \\ China \\ ${ }^{4}$ Huaibei Miner's General Hospital, Huaibei, Anhui Province, China \\ *These authors contributed equally to this study. \\ Corresponding author: Z. Li \\ E-mail: stronglz110732@163.com
}

Genet. Mol. Res. 14 (4): 17496-17504 (2015)

Received August 22, 2015

Accepted October 6, 2015

Published December 21, 2015

DOI http://dx.doi.org/10.4238/2015.December.21.21

\begin{abstract}
Enterovirus 71 (EV71) and coxsackievirus A16 (CA16) have been the primary causative agents of hand, foot, and mouth disease (HFMD) outbreaks in mainland China in the past. Hence, the surveillance of HFMD has mostly focused on these viruses. However, in recent years, coxsackievirus A10 (CA10) has also been associated with the increasing sporadic HFMD cases and outbreaks. Therefore, a sensitive assay for rapid detection of the CA10 RNA is necessary for disease control. Here, we have developed a specific TaqMan real-time RT-PCR assay by analyzing VP1 gene sequences of CA10 strains from different locations. The assay has been shown to be specific, sensitive, and robust through detection of other related viruses, standard curves, and clinical samples, respectively. This is the first report on development of a VP1 gene-based TaqMan realtime RT-PCR assay for rapid diagnosis of CA10 virus.
\end{abstract}

Key words: CA10; HFMD; TaqMan real-time RT-PCR; China 


\section{INTRODUCTION}

Hand, foot, and mouth disease (HFMD) is a common disease in children, which is characterized by mild fever along with cutaneous vesicular rashes on the palms, soles, buttocks, and mouth (oropharyngeal ulcers) (McMinn et al., 2002; Shah et al., 2003). Several large epidemics of HFMD have been reported in the Asia-Pacific region, especially in Southeast Asia. Previously, HFMD was predominantly caused by enterovirus 71 (EV71) and coxsackievirus A16 (CA16) (Zhang et al., 2009; Zhang et al., 2010; Lu et al., 2012), as well as other enteroviruses such as CA2, CA4, CA5, CA6, CA8 and CA10 (Blomqvist et al., 2010). Among these, increased incidences of CA10associated HFMD epidemics have been reported around the world (Itagaki et al., 1983; He et al., 2013). Previously, CA10 had been isolated from in sporadic HFMD cases in New Zealand in 1957 and the first clustering outbreak of HFMD caused by CA10 was reported in Japan in 1983 (Seddon et al., 1971; Itagaki et al., 1983). CA10 accounted for $11.8 \%$ of all cases in the largest HFMD outbreak in Singapore in 2008 (Wu et al., 2010) and was one of the main pathogens in the Finland outbreak, which occurred around the same time (Blomqvist et al., 2010). A sentinel surveillance study in France in 2010 showed that the predominant serotype was CA10 (39.9\%), whereas the infection rate of EV71 and CA16 was low (23.8\%) (Mirand et al., 2012). An onychomadesis outbreak reported in 2008 in Spain was shown to be caused by CA10 (Davia et al., 2010). CA10, as the third serotype, caused outbreaks of HFMD in Taiwan in 2005 (Tseng et al., 2007).

In mainland China, CA10 and CA6 were known to be co-circulating with EV71 and CA16 (Lu et al., 2012; He et al., 2013). Recently, CA10 has emerged as a key pathogen in the outbreaks of HFMD in mainland China in addition to EV71 and CA16. A clustering outbreak of HFMD caused by CA10 was reported in Wendeng City of Shandong province in 2009 (Xing et al., 2014). All these studies are strongly suggestive of CA10 infection as an important cause of HFMD. Therefore, an effective detection method for CA10 is imperative to strengthen the surveillance of CA10 infection in HFMD epidemics and control the outbreaks.

This study aims at developing a sensitive and specific real-time RT-PCR assay for the rapid detection of CA10, which is not only simple and effective in clinical diagnosis but also more advantageous than the commercial kits available in the domestic market.

\section{MATERIAL AND METHODS}

\section{Specimen collection}

Specimens from 133 children clinically diagnosed with HFMD were collected between 2011 and 2012 at the Second People's Hospital of Fuyang City, Anhui Province, located in the middle east of China. Pharyngeal swabs from 72 boys and 61 girls aged between 1 month and 6 years were obtained with their parents' consent. The samples were analyzed at the diagnostic virology laboratory of Fuyang City Centers for Disease Control and Prevention (CDC).

Viruses including enteroviruses such as CA10, CA16, EV71, CB2, CA4, and Poliovirus type I-III as well as others such as VZV Oka vaccine, Rubella Virus (RV), Measles virus (MV), ECHO-5, HSV-1, HSV-2, Japanese Encephalitis Vaccine (JE vaccine), all of which were available in our laboratory collection, were used for reference.

\section{Design and evaluation of primers and TaqMan probe}

Primers and a Taqman probe targeting one of the highly conserved regions of VP1 gene 
were designed by analyzing all the CA10 nucleotide sequences available in GenBank using the Primer Premier 5.0 software (Table 1) and synthesized by Invitrogen Co. (Invitrogen, Shanghai, China). The primers were validated by identifying the VP4 gene (Ishiko et al., 2002).

Table 1. Primers and probe from VP1 gene of CA10 for diagnostic real-time RT-PCR assay.

\begin{tabular}{llcc}
\hline Primers/probe & Sequence $\left(5^{\prime} \rightarrow 3^{\prime}\right)$ & Nucleotide position & Size of amplicon \\
\hline CA10F & GAAATGGRGTGTTGGAAACCA & $2674-2654$ & 148 bp \\
CA10R & TTTCTGCGRAGTTGGACAAAG & $2801-2781$ & \\
CA10P & FAM-ATCAACCAYTTCTTCTCYCGCTCTGG-TAMRA & $2702-2677$ & \\
\hline
\end{tabular}

\section{CA10 target gene cloning}

CA10 virus was isolated from Fuyang city and cultured in RD cells, the genomic RNA extracted using TRIzol rReagent (Invitrogen), then and reverse transcription transcribed. The target gene segment was amplified using the QIAGEN One-Step RT-PCR Kit (Qiagen, Germany). The reaction mixture $(50 \mu \mathrm{L})$ contained $10 \mu \mathrm{L} 5 \mathrm{X}$ One-Step RT-PCR Buffer, $2 \mu \mathrm{L}$ dNTP Mix, $3 \mu \mathrm{L}$ each CA10F and CA10R primers respectively, $2 \mu \mathrm{L}$ One-Step RT-PCR Enzyme Mix, $0.2 \mu \mathrm{L}$ RNase inhibitor, and $5 \mu \mathrm{L}$ template RNA. Reverse transcription was carried out at $50^{\circ} \mathrm{C}$ for $30 \mathrm{~min}$. PCR parameters were set at $95^{\circ} \mathrm{C}$ for $15 \mathrm{~min}$ for the initial activation, followed by 35 cycles of $94^{\circ} \mathrm{C}$ for $30 \mathrm{~s}, 55^{\circ} \mathrm{C}$ for 30 $\mathrm{s}$, and $72^{\circ} \mathrm{C}$ for $10 \mathrm{~s}$. The PCR product was cloned into PCR 2.1 Vector (Life Technologies, Shanghai, China) and the resulting PCR2.1-T-VP1 was confirmed by sequencing.

\section{Analyses of the specificity and sensitivity of the real-time RT-PCR assay}

TaqMan real-time RT-PCR was performed by using the One Step PrimeScript RT-PCR Kit (TaKaRa, Dalian, China) following the manufacturer protocols. The sensitivity of the real-time RT-PCR assay was assessed by the detection range obtained using 10-fold serial dilutions of the recombinant plasmid (PCR2.1-T-VP1) as templates. In order to evaluate the specificity of the assay, RNA from reference viruses namely CA16, EV71, CB2, CA4, Sabin1, 2, and 3, VZV, RV, MV, ECHO5, HSV-1, HSV-2, and JE vaccine were amplified. The recombinant plasmid (PCR2.1-T-VP1) was used as positive control. All reactions were performed on an $\mathrm{ABI} 7500$ sequence detection system.

\section{Determination of detection and error rates of the assay}

To determine the detection rate of the real-time RT-PCR assay, RNA samples from 8 CA10 clinical isolates obtained from Fuyang City collected during 2009-2010 were used as templates. These were previously identified by the amplification and sequencing of VP4 gene (Ishiko et al., 2002; Zhao et al., 2013). Further, to evaluate the error rate and false positives, 26 throat swabs from HFMD known to be unrelated to EV71 or CA16 were analyzed by the TaqMan real-time RTPCR assay. Products of positive samples were then purified by agarose gel (1.2\%) electrophoresis and sequenced for confirmation.

\section{RESULTS}

\section{Sequence analysis of VP1 gene}

VP1 gene sequences of CA10 virus from GenBank database as well as those of the 
8 isolates from Fuyang city (FY01-08) were analyzed for nucleotide sequence homology and a dendrogram was constructed (Figure 1) by using the Mega 5.2 software (Biodesign Institute, AZ, USA) to estimate the genetic distances. The GenBank accession numbers of the sequences included in the analysis are: AB739052.1 (YunNan, China); KC834855.1-KC834877.1, JX569076.1 (ShangHai, China); JQ320248.1, JQ320252.1 (XiaMen, China); GU947774.1-GU947787.1, GQ214172.1-GQ214177.1 (ShanDong, China); JX154970.2-JX154982.2, JX473446.2, JX473447.2, JX473449.2, JX473462.2, KC867018.1-KC867044.1 (ShenZhen, China); FuYang01FuYang08 (Unregistered sequences, Fuyang, China); JN896787.2, JN896805.1, JN896804.1, JN896799.1, JN896798.1, JN896789.1, JN896784.1 JN896779.1 (TaiWan); HE573016.1, HE572941.1-HE572992.1 (France).

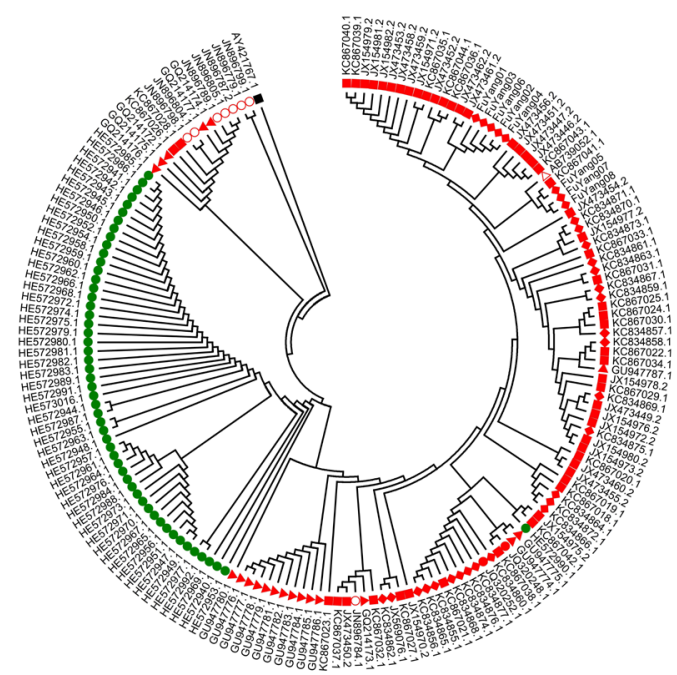

Figure 1. Phylogenetic analysis of the partial VP1 (nt2642-nt2798) gene of CA10 virus. Evolutionary analysis was conducted with 171 nucleotide sequences using the MEGA 5.2 software and evolutionary history inferred using the neighbor-joining method.

While the CA10 Kowalik strain branched separately, CA10 isolates from France and mainland China were distributed over two different clusters. Furthermore, CA10 isolates from the different regions in mainland China could be divided into different subtypes. Primers and the Taqman probe were designed from the region that was conserved across the CA10 VP1 gene sequences (Figure 2).

\section{Cloning of VP1 gene fragment and evaluation of primers and probe}

The conserved VP1 gene fragment from CA10 isolates (virus strain supplied by the diagnostic virology laboratory of Fuyang City Centers for Disease Control and Prevention) were amplified by RT-PCR, and the product was purified, cloned into PCR $2.1 \mathrm{vVector}$ and sequenced. The nucleotide sequence of the resulting VP1 fragment had higher homology to isolates from mainland China than those from France and Finland. Several substitutions were found in the sequence of the segment; however, they had little impact on the primers and probe (Figure 2).

The nucleotide sequences of the primers and Taqman probe were searched against 
sequence databases by using the BLASTn tool (National Center for Biotechnology Information of $\mathrm{NIH}$ ) and none of them showed any significant homology that may yield non-specific amplification or hybridization (data not shown).

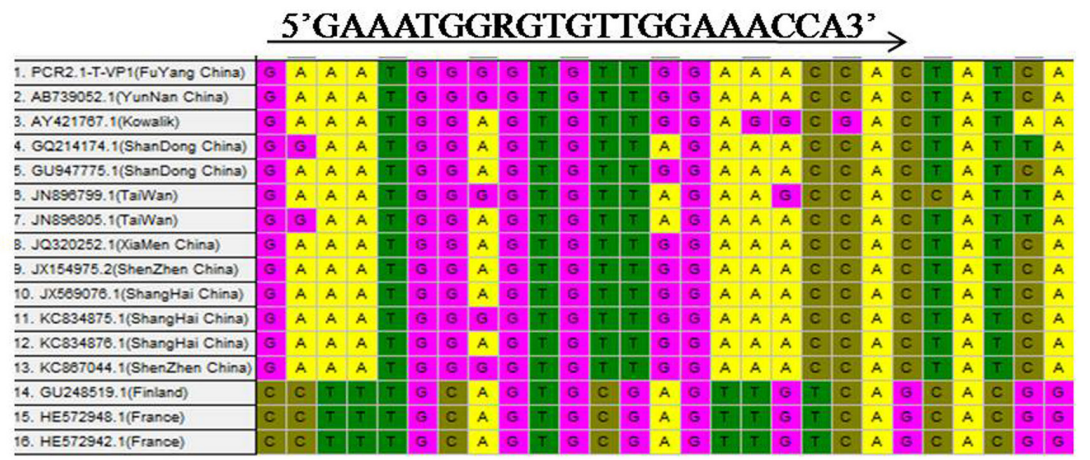

\section{5'FAM-ATCAACCAYTTCTTCTCYCGCTCTGG- TAMRA 3'}
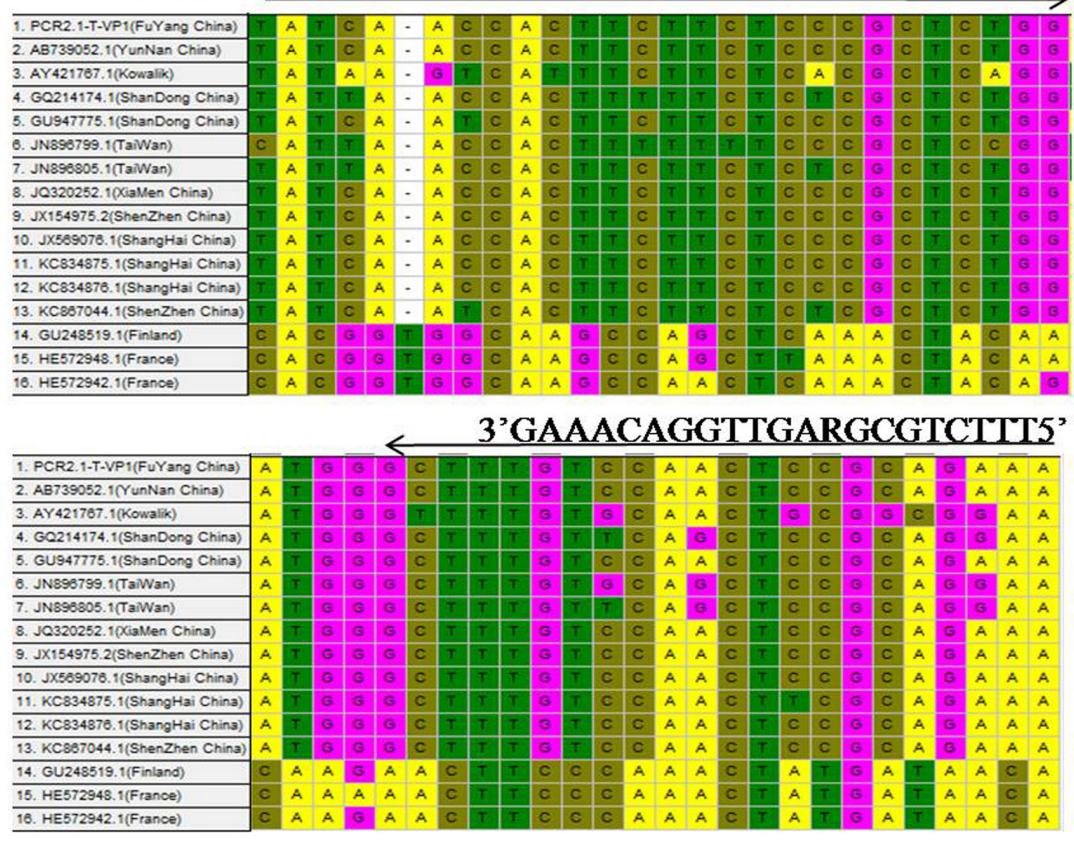

Figure 2. Homology analysis of the partial VP1 gene across CA10 strains. The primers and Taqman probe were designed based on the conserved nucleotides in the VP1 gene of different strains of CA10.

\section{Specificity and sensitivity of the TaqMan real-time RT-PCR assay}

In order to test the CA10 specificity of the assay, genomic nucleic acid from reference viruses including enteroviruses (EV71, CA4, CA16, CB2, Poliovirus type I-III), and others such as VZV Oka vaccine, RV, MV, ECHO-5, HSV-1, HSV-2, and JE vaccine were analyzed by the real-time RT-PCR assay. None of the reference viruses yielded any positive fluorescence signal (Figure 3A). 

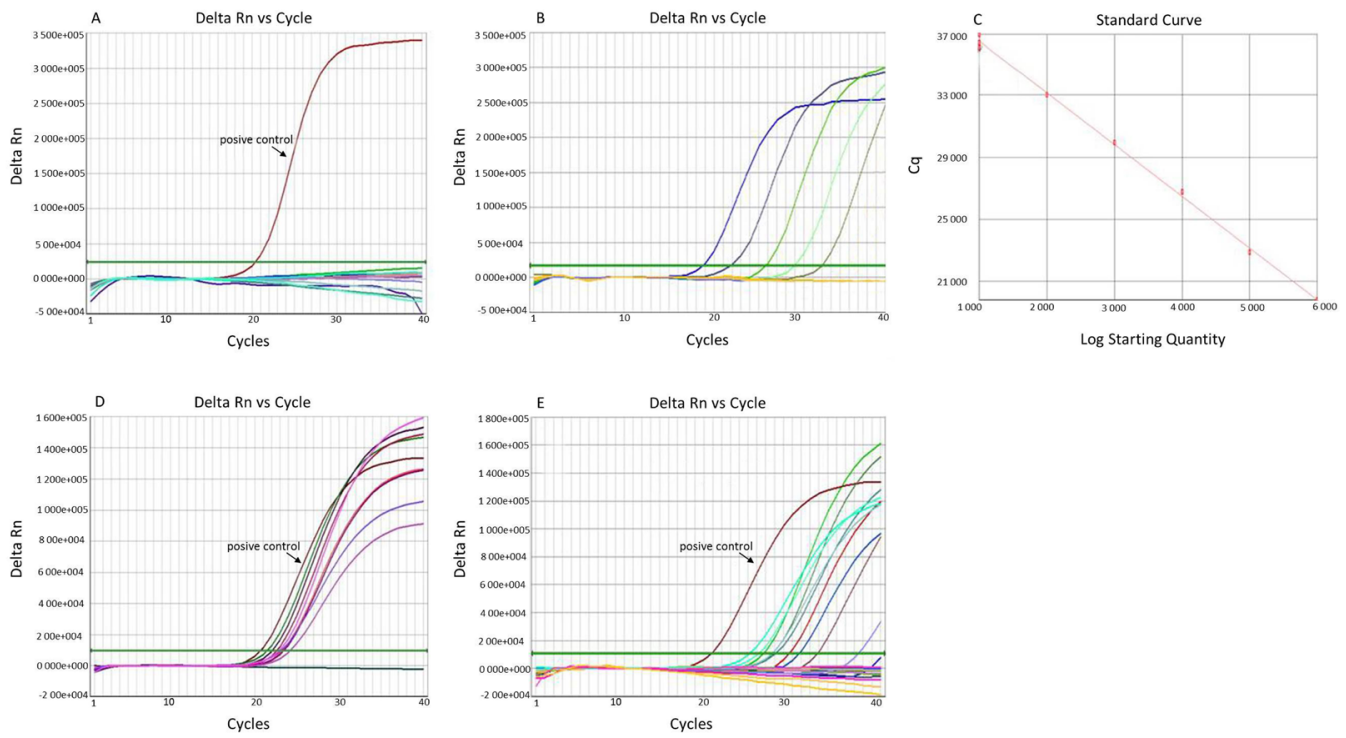

Figure 3. Sensitivity and specificity analyses of the real-time RT-PCR assay and detection of clinical samples. A. Amplification of reference viruses and vaccine templates using the CA10-specific real-time RT-PCR to evaluate the assay specificity. The recombinant plasmid (PCR2.1-T-VP1) was used as positive control. B. Standard curve generated using serial dilutions of recombinant plasmids PCR2.1-T-VP1 (10-105 copies/ $\mu \mathrm{L})$ as templates to establish the detection limits of the assay. The values are representative of three replicates. C. Typical amplification plot of serial dilutions of PCR2.1-T-VP1 (R2 = 0.9981). D. The detectable rate of real-time RT-PCR assay was identified by using eight isolates of CA10, which turned out to be $100 \%$. E. Twenty-six clinical HFMD samples (non-EV71 and non-CA16) tested by the CA-10 specific diagnostic assay. The recombinant plasmid PCR2.1-T-VP1 was used as control.

Serial dilutions of the recombinant plasmid PCR2.1-T-VP1 (10-105 copies $/ \mu L)$ were used to determine the detection limits of the assay. The lowest detected concentration was 10 copies/ $\mu \mathrm{L}$ and a linear plot was obtained with increasing concentration (Figure $3 \mathrm{~B}$ ). The amplification efficiency of PCR2.1-T-VP1 at different concentrations was highly similar $\left(R^{2}>0.99\right)$ (Figure 3C).

\section{Verification of the assay using clinical specimens}

CA10 clinical isolates and non-EV71 and non-CA16 specimens were used to estimate the diagnostic ability of the assay. All the eight tested CA10 isolates from Fuyang city were detected and their detection rate was 100\%, same as the positive control (Figure 3D). Subsequently, 26 throat swabs from patients with HFMD that was not caused by EV71 and CA16 were tested as templates using the CA10-specific TaqMan real-time RT-PCR assay, which resulted in 10 positive and 16 negative samples (Figure $3 E$ ). In order to verify these results, the PCR products (partial VP1 gene) of all the CA10-positive samples were purified by using agarose gel (1.2\%) electrophoresis and verified by sequencing (Figure 4). Furthermore, VP4 gene from the CA10 positive samples were amplified and verified by sequencing. The results demonstrated that the concordance rate between the two assays was $100 \%$ (data not shown). 


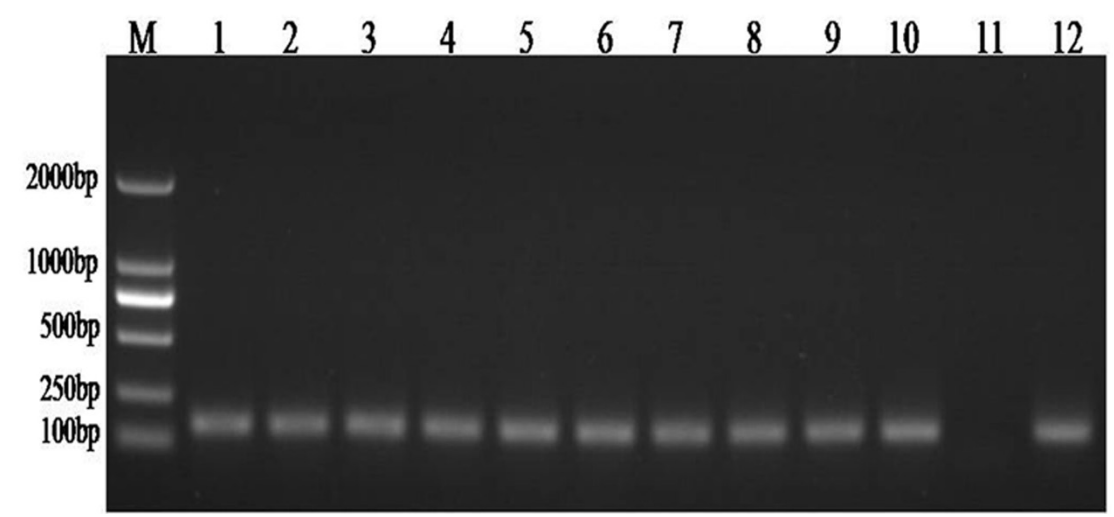

Figure 4. Verification of CA10 strains detected from the clinical samples. Lane M: DL2000 marker; lanes 1-10: PCR products of the CA10-positive clinical samples; lanes 11 and 12: negative control and positive control visualized on an agarose gel. All bands are of the expected size (148 bp).

\section{DISSCUSSION}

Human enteroviruses (HEVs) comprise more than 90 serotypes, which cause a wide spectrum of acute febrile diseases, especially HFMD among infants and children. Different causative pathogens of HFMD such as EV71, CA16, CA10, and CB2 have been reported so far (Blomqvist et al., 2010). The two major causative agents of HFMD, EV71 and CA16 were first isolated in Canada and USA in 1958 and 1969, respectively (Zhu et al., 2010). In mainland China, clinical diagnoses and surveillance mainly targeted detection of EV71 and CA16 using specific primers. In contrast, other HEVs were identified by universal primers. Although these primers were reported to characterize unknown HEVs (Ishiko et al., 2002; Nix et al., 2006; Oberste et al., 2006; $\mathrm{Ji}$ et al., 2012), this method requires independent clones to be selected for sequencing or direct sequencing of PCR products, both of which may be result in some pathogens left undetected. Hence, universal primers for HEVs are not reliable for rapid clinical diagnoses.

Real-time RT-PCR assays developed in recent years possess several advantages such as improved sensitivity, specificity, and reproducibility, and have been employed to detect a range of respiratory viruses (van Elden et al., 2001; Emery et al., 2004; Cui et al., 2013). This study aimed at developing a real-time RT-PCR assay for rapid detection and surveillance of CA10, which is specific, sensitive and quantitative compared to the conventional assay using HEV universal primers. In this CA10-specific real-time RT-PCR assay, FAM and TAMRA were used in the TaqMan probe. In addition, our results showed high sensitivity of the assay for CA10 with detection limit as low as 10 copies/ $\mu \mathrm{L}$ of CA10 template. For CA10 strains accounted for $38 \%$ of the non-EV71 and non-CA16 HFMD clinical specimens tested using the diagnostic assay, which was verified by using nest PCR to check VP4 gene (Zhao et al., 2013).

Nevertheless, Figures 1 and 2 indicate that VP1 gene of CA10 isolates were regionspecific as known from the previous studies (Yang et al., 2010; Lu et al., 2012; Mirand et al., 2012; Xing et al., 2014). Therefore, the real-time RT-PCR system seems to be limited to effective detection of CA10 strains isolated from France and Finland. A multiplex real-time RT-PCR system for rapid diagnosis and surveillance of global CA10 strains may be the best solution. However, the TaqMan real-time RT-PCR system established in this study will still be a very useful method for rapid detection of CA10 in mainland China. 


\section{Conflicts of interest}

The authors declare no conflict of interest.

\section{ACKNOWLEDGMENTS}

The authors would like to acknowledge all the participants for providing clinical samples. We thank the National Nature Science Foundation of China (\#81302701), the Grants for Scientific Research of BSKY (\#XJ201226) from Anhui Medical University, and the Natural Science Foundation of Anhui Province (\#1308085QC53) for financially supporting this study.

\section{REFERENCES}

Blomqvist S, Klemola P, Kaijalainen S, Paananen A, et al. (2010). Co-circulation of coxsackieviruses A6 and A10 in hand, foot and mouth disease outbreak in Finland. J. Clin. Virol. 48: 49-54.

Cui A, Xu C, Tan X, Zhang Y, et al. (2013). The development and application of the two real-time RT-PCR assays to detect the pathogen of HFMD. PLoS One 8: e61451.

Davia JL, Bel PH, Ninet VZ, Bracho MA, et al. (2011). Onychomadesis outbreak in Valencia, Spain associated with Hand, foot, and mouth disease caused by enteroviruses. Pediatr. Dermatol. 28: 1-5.

Emery SL, Erdman DD, Bowen MD, Newton BR, et al. (2004). Real-time reverse transcription-polymerase chain reaction assay for SARS-associated coronavirus. Emerg. Infect. Dis. 10: 311-316.

He YQ, Chen L, Xu WB, Yang H, et al. (2013). Emergence, circulation and spatiotemporal phylogenetic analysis of Coxsackievirus A6- and Coxsackievirus A10-associated hand, foot and mouth disease infections from 2008 to 2012 in Shenzhen, China. J. Clin. Microbiol. 51: 3560-3566.

Ishiko H, Shimada Y, Yonaha M, Hashimoto O, et al. (2002). Molecular diagnosis of human enteroviruses by phylogeny-based classification by use of the VP4 sequence. J. Infect. Dis. 185: 744-754.

Itagaki A, Ishihara J, Mochida K, Ito Y, et al. (1983). A clustering outbreak of hand, foot, and mouth disease caused by coxsackievirus A10. Microbiol. Immunol. 27: 929-935.

Ji Z, Wang X, Zhang C, Miura T, et al. (2012). Occurrence of hand-foot-and-mouth disease pathogens in domestic sewage and secondary effluent in Xi'an, China. Microbes Environ. 27: 288-292.

Lu QB, Zhang XA, Wo Y, Xu HM, et al. (2012). Circulation of Coxsackievirus A10 and A6 in hand-foot-mouth disease in China, 2009-2011. PLoS One 7: e52073.

McMinn PC (2002). An overview of the evolution of enterovirus 71 and its clinical and public health significance. FEMS Microbiol. Rev. 26: 91-107.

Mirand A, Henquell C, Archimbaud C, Ughetto S, et al. (2012). Outbreak of hand, foot and mouth disease/herpangina associated with coxsackievirus A6 and A10 infections in 2010, France: a large citywide, prospective observational study. Clin. Microbiol. Infect. 18: E110-118.

Nix WA, Oberste MS and Pallansch MA (2006). Sensitive, seminested PCR amplification of VP1 sequences for direct identification of all enterovirus serotypes from original clinical specimens. J. Clin. Microbiol. 44: 2698-2704.

Oberste MS, Maher K, Williams AJ, Dybdahl-Sissoko N, et al. (2006). Species-specific RT-PCR amplification of human enteroviruses: a tool for rapid species identification of uncharacterized enteroviruses. J. Gen. Virol. 87: 119-128.

Saitou N and Nei M (1987). The neighbor-joining method: a new method for reconstructing phylogenetic trees. Mol. Biol. Evol. 4: $406-425$.

Seddon JH and Duff MF (1971). Hand-foot-and-mouth disease: Coxsackie virus types A5, A10, and A16 infections. N. Z. Med. J. 74: 368-373.

Shah VA, Chong CY, Chan KP, Ng W, et al. (2003). Clinical characteristics of an outbreak of hand, foot and mouth disease in Singapore. Ann. Acad. Med. Singapore 32: 381-387.

Tseng FC, Huang HC, Chi CY, Lin TL, et al. (2007). Epidemiological survey of enterovirus infections occurring in Taiwan between 2000 and 2005: analysis of sentinel physician surveillance data. J. Med. Virol. 79: 1850-1860.

van Elden LJ, Nijhuis M, Schipper P, Schuurman R, et al. (2001). Simultaneous detection of influenza viruses A and B using real-time quantitative PCR. J. Clin. Microbiol. 39: 196-200.

Wu Y, Yeo A, Phoon MC, Tan EL, et al. (2010). The largest outbreak of hand; foot and mouth disease in Singapore in 2008: the role of enterovirus 71 and coxsackievirus A strains. Int. J. Infect. Dis. 14: e1076-1081. 
Xing W, Liao Q, Viboud C, Zhang J, et al. (2014). Hand, foot, and mouth disease in China, 2008-12: an epidemiological study. Lancet Infect. Dis. 14: 308-318.

Yang H, Tao ZX, Wang HY, Li Y, et al. (2010). The genetic characterization of VP1 region of coxsackie virus A10 isolated from hand, foot and mouth disease cases in Shandong Province of China. Chin. J. Infect. Dis. 28: 385-389.

Zhang Y, Tan XJ, Wang HY, Yan DM, et al. (2009). An outbreak of hand, foot, and mouth disease associated with subgenotype C4 of human enterovirus 71 in Shandong, China. J. Clin. Virol. 44: 262-267.

Zhang Y, Zhu Z, Yang W, Ren J, et al. (2010). An emerging recombinant human enterovirus 71 responsible for the 2008 outbreak of hand foot and mouth disease in Fuyang city of China. Virol. J. 7: 94.

Zhao L, Chen JX and Wang ML (2013). Gene characteristics analysis for CoxA10 VP4 region of hand-foot-mouth disease in Fuyang. Chin. J. Dis. Control Prev. 17: 480-483.

Zhu Z, Zhu S, Guo X, Wang J, et al. (2010). Retrospective seroepidemiology indicated that human enterovirus 71 and coxsackievirus A16 circulated wildly in central and southern China before large-scale outbreaks from 2008. Virol. J. 7: 300. 